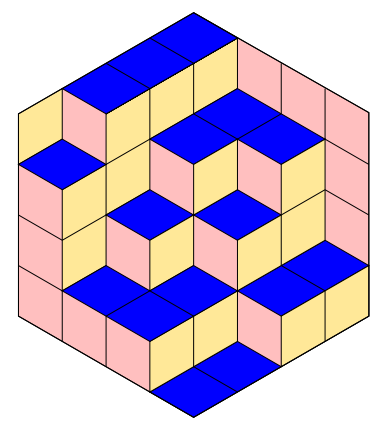

ALGEBRAIC COMBINATORICS

Harvey I. Blau

On residually thin and nilpotent table algebras, fusion rings, and association schemes Volume 5, issue 1 (2022), p. 21-36.

https: //doi.org/10.5802/alco.194

(C) The journal and the authors, 2022.

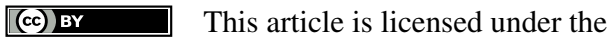

Creative Commons AtTribution 4.0 International LiCEnSE.

http://creativecommons.org/licenses/by/4.0/

Access to articles published by the journal Algebraic Combinatorics on the website http://alco.centre-mersenne.org/ implies agreement with the Terms of Use (http://alco.centre-mersenne.org/legal/).

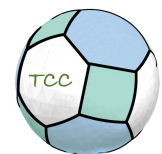

Algebraic Combinatorics is published by The Combinatorics Consortium and is a member of the Centre Mersenne for Open Scientific Publishing

www.tccpublishing.org

www.centre-mersenne.org 


\title{
On residually thin and nilpotent table algebras, fusion rings, and association schemes
}

\author{
Harvey I. Blau
}

\begin{abstract}
Residually thin and nilpotent table algebras, which are abstractions of fusion rings and adjacency algebras of association schemes, are defined and investigated. A formula for the degrees of basis elements in residually thin table algebras is established, which yields an integrality result of Gelaki and Nikshych as an immediate corollary; and it is shown that this formula holds only for such algebras. These theorems for table algebras specialize to new results for association schemes. Bi-anchored thin-central (BTC) chains of closed subsets are used to define nilpotence, in the manner of Hanaki for association schemes. Lower BTC-chains are defined as an abstraction of the lower central series of a finite group. A partial characterization is proved; and a family of examples illustrates that unlike the case for finite groups, there is not necessarily a unique lower BTC-chain for a nilpotent table algebra or association scheme.
\end{abstract}

\section{INTRODUCTION}

We explore two related aspects of some important algebraic and combinatorial structures: namely, the properties called residual thinness and nilpotence. The former concept has been studied in the framework of association schemes by Zieschang $[13,14]$ and Hanaki and Shimabukuro [11], among others; and for hypergroups (as an algebraic generalization of schemes) by French and Zieschang [7]. It has been analyzed (evidently independently) in the setting of fusion categories and fusion rings by Gelaki and Nikshych [8]. They use the term "nilpotent" for what the other authors above call "residually thin". This usage has the desirable consequence that a finite group is nilpotent in the classical sense if and only if its representation category (resp. character ring) is nilpotent as a fusion category (resp. fusion ring) [8, Remark 4.7]. However, the group algebra of any finite group, as a fusion ring, is nilpotent according to their definition. This seems to leave room for an alternative definition of nilpotent. The one presented in this paper (see Definition 1.3 below) is a direct generalization of the one given for association schemes by Hanaki [9].

Our context here is the family of table algebras, finite dimensional algebras over the complex numbers with a distinguished basis that satisfies certain axioms (see Definition 1.1 below). The adjacency (or Bose-Mesner) algebras of association schemes, group algebras, and Hecke (double coset) algebras constructed from group algebras are examples, and fusion rings comprise a subfamily. Hypergroups, in the sense of [7], are generalizations of table algebras. Our main results include a formula for the degrees of the basis elements of a residually thin table algebra, and a proof that this formula

Manuscript received 5th September 2020, revised 10th August 2021, accepted 16th August 2021.

Keywords. Table algebra, fusion ring, association scheme, residually thin, nilpotent, thin central chain. 
holds only for such algebras (see Theorem 1.7 below). Gelaki and Nikshych's integrality theorem [8, Corollary 5.3] for fusion categories is an immediate consequence. These are evidently new results for association schemes as well. We also define the notion of thin central chains for a table algebra, in particular upper, lower, and bi-anchored such chains, and use it to exposit our alternate definition of nilpotent table algebra. This definition coincides with the usual one in the case of a finite group; and when applied to commutative table algebras, it is equivalent to both the author's [2] and Gelaki and Nikshych's [8] definitions. Theorems 1.14 and 1.20 below relate residual depth to nilpotence class (see Definitions 1.15, 1.21), and thus extend Gelaki and Nikshych's result for the commutative case [8, Theorem 4.16]. Lower bi-anchored thin-central chains exist for any nilpotent table algebra by Definition 1.11. Theorem 1.23 gives a partial characterization of them. But unlike the case of the lower central series of a finite group, they are not necessarily unique. Example 5.5 shows this, and thereby gives a negative answer to a question of Hanaki [9, Question 2.11] regarding uniqueness of lower central series in an association scheme.

We recall a few well known definitions and facts needed in order to state the main results. These are developed in a number of sources, in particular $[1,6,3]$.

Definition 1.1. A table algebra $(T A)(A, B)$ is a finite dimensional algebra $A$ over the complex numbers $\mathbb{C}$, and a distinguished basis $B$ that contains $1_{A}$, such that the following properties hold:

(1a) The structure constants for $B$ are all nonnegative real numbers; that is, for all $b, c \in B$,

$$
b c=\sum_{d \in B} \lambda_{b c d} d, \quad \text { for some } \lambda_{b c d} \in \mathbb{R}_{\geqslant 0} .
$$

(1b) There is an algebra anti-automorphism (denoted by ${ }^{*}$ ) of $A$ such that $\left(a^{*}\right)^{*}=a$ for all $a \in A$; and $B^{*}=B$.

(1c) For all $b, c \in B, \lambda_{b c 1}=0$ if and only if $c \neq b^{*}$.

It follows as a consequence of the definition that $\lambda_{b b^{*} 1}=\lambda_{b^{*} b 1}>0$ for all $b \in B$. Frobenius-Perron eigenvalue theory yields that for each table algebra there exists a unique algebra homomorphism $\delta: A \rightarrow \mathbb{C}$, called the degree map, such that $\delta(b)=$ $\delta\left(b^{*}\right)>0$ for all $b \in B$. The table algebra $(A, B)$ is called standard if for all $b \in B$, $\delta(b)=\lambda_{b b^{*} 1}$. Any table algebra can be rescaled (replace each $b \in B$ by $\beta_{b} b$, for suitable $\left.\beta_{b}>0\right)$ to one that is standard.

Let $(A, B)$ be a standard table algebra (STA). For any subsets $S, T$ of $B$, the set product $S T:=\cup_{s \in S, t \in T} \operatorname{Supp}_{B}(s t), S^{*}:=\left\{s^{*} \mid s \in S\right\}$, and $S^{+}:=\sum_{s \in S} s$. Note that set product is associative. If $T=\{t\}$, a singleton set, then $S\{t\}$ (resp. $\{t\} S, S\{t\} S$ ) is denoted $S t$ (resp. $t S, S t S$ ). The order of a subset $S$ is $o(S):=\delta\left(S^{+}\right)$.

A nonempty subset $C \subseteq B$ is called closed if $C^{*} C \subseteq C$. In this case, $(\mathbb{C} C, C)$ is again a table algebra, and the set of left cosets $C b$ for $b \in B$ partition $B$, as do the right cosets $b C$, and the $C$ - $C$ double cosets $C b C$. A quotient element, for any $b \in B$, is $b / / C:=o(C)^{-1}(C b C)^{+}$. Let $B / / C:=\{b / / C \mid b \in B\}$, and $A / / C:=\mathbb{C}(B / / C)$. Then $(A / / C, B / / C)$ is a STA, called the quotient algebra, or double coset algebra of $(A, B)$ by the closed subset $C$. Its degree map is $\delta \downarrow_{A / / C}$, and its anti-automorphism is ${ }^{*} \downarrow_{A / / C}$. Furthermore, $o(B / / C)=o(B) / o(C)$. The closed subsets $D$ with $C \subseteq D \subseteq B$ are in bijection with the closed subsets of $B / / C$ via $D \leftrightarrow D / / C$ (see Proposition 2.5 below). The closed subset $C$ is called normal (resp. strongly normal) in $B$ if $b C=C b$ (resp. $b C b^{*}=C$ ) for all $b \in B$. Strongly normal closed subsets are normal, but the converse is not always true.

An element $x \in B$ is called thin (or linear, or grouplike) if $x x^{*}=1$. This is equivalent to $\delta(x)=1$; and if $x$ is thin, then $x b \in B$ and $b x \in B$ for all $b \in B$. 
So $\delta(x b)=\delta(b x)=\delta(b)$ for all $b \in B$. Now $O_{\vartheta}(B):=\left\{x \in B \mid x x^{*}=1\right\}=\{x \in$ $B \mid \delta(x)=1\}$ is a group, called the thin radical of $B$; and $B$ is called thin if $B=O_{\vartheta}(B)$. Obviously, $O_{\vartheta}(B)$ is the unique maximal closed subset $D$ of $B$ such that $D$ is thin. For $C$ a closed subset of $B$, the quotient $B / / C$ is thin if and only if $C$ is strongly normal in $B$. Since the intersection of strongly normal closed subsets is again strongly normal, there is a unique minimal closed subset of $B$, denoted $O^{\vartheta}(B)$, such that $B / / O^{\vartheta}(B)$ is thin. Now $O^{\vartheta}(B)$ is called the thin residue of $B$, and it equals the closed subset of $B$ generated by $\operatorname{Supp}_{B}\left(b b^{*}\right)$ for all $b \in B$. (See [13, Theorem 2.3.1] or [14, Theorem 3.2.1], where the algebraic proof for association schemes holds verbatim for table algebras.)

Throughout, $Z(A)$ will denote the center of the algebra $A$, and $Z(B)$ will mean $B \cap Z(A)$.

Definition 1.2. A chain $\mathcal{C}$ of length $n$ is a collection $\left\{C_{i}\right\}_{i=0}^{n}$ of closed subsets of $B$ such that either $C_{0} \subset C_{1} \subset \cdots \subset C_{n-1} \subset C_{n}$ or $C_{n} \subset C_{n-1} \subset \cdots \subset C_{1} \subset C_{0}$. It is called a bi-anchored chain (B-chain) if $C_{0}=\{1\}$ and $C_{n}=B$ (or $C_{n}=\{1\}$ and $C_{0}=B$ ). It is a thin chain (T-chain) if $C_{i+1} / / C_{i}$ is thin for all $0 \leqslant i \leqslant n-1$ (or $C_{i-1} / / C_{i}$ is thin for all $\left.1 \leqslant i \leqslant n\right)$. It is called a thin-central chain (TC-chain) if $C_{i+1} / / C_{i}$ is thin and $C_{i+1} / / C_{i} \subseteq Z\left(B / / C_{i}\right)$ for all $0 \leqslant i \leqslant n-1$ (or $C_{i-1} / / C_{i}$ is thin and $C_{i-1} / / C_{i} \subseteq Z\left(B / / C_{i}\right)$ for all $\left.1 \leqslant i \leqslant n\right)$.

DEFINITION 1.3. A STA $(A, B)$ is residually thin if there exists a bi-anchored thin chain (BT-chain). It is nilpotent if there exists a bi-anchored thin-central chain (BTCchain).

REMARK 1.4. It is immediate from the definition that for any finite group $G$, the group algebra $(\mathbb{C} G, G)$ is nilpotent as a STA if and only if $G$ is nilpotent in the usual group-theoretic sense.

Proposition 1.5. Let $(A, B)$ be a residually thin $S T A$, with BT-chain $B=C_{0} \supset$ $C_{1} \supset \cdots \supset C_{n-1} \supset C_{n}=\{1\}$. Then $o\left(C_{i}\right)$ is an integer, and $o\left(C_{i+1}\right) \mid o\left(C_{i}\right)$ for all $0 \leqslant i \leqslant n-1$.

Proof. Since each $C_{j} / / C_{j+1}$ is a group, o $\left(C_{j} / / C_{j+1}\right)=o\left(C_{j}\right) / o\left(C_{j+1}\right)$ is an integer for $0 \leqslant j \leqslant n-1$. Since $o\left(C_{i}\right)=o\left(C_{i+1}\right) o\left(C_{i} / / C_{i+1}\right)=\prod_{j \geqslant i} o\left(C_{j} / / C_{j+1}\right)$, the result follows.

Definition 1.6. Let $(A, B)$ be a STA with a $B$-chain $\mathcal{C}: B=C_{0} \supset C_{1} \supset \cdots C_{n-1} \supset$ $C_{n}=\{1\}$. For any $1 \leqslant i \leqslant n$ and any $b \in B \backslash C_{i}$, define the positive integer $m(\mathcal{C}, i, b)$ for $i<n$ by

$$
m(\mathcal{C}, i, b):=\prod_{j=i}^{n-1} \operatorname{card}\left(C_{j} / / C_{j+1} \cdot b / / C_{j+1} \cdot C_{j} / / C_{j+1}\right),
$$

where $C_{j} / / C_{j+1} \cdot b / / C_{j+1} \cdot C_{j} / / C_{j+1}$ is the $C_{j} / / C_{j+1}-C_{j} / / C_{j+1}$ double coset of $b / / C_{j+1}$ in $B / / C_{j+1}$; and $m(\mathcal{C}, n, b):=\operatorname{card}\left(C_{n} b C_{n}\right)=\operatorname{card}\{b\}=1$.

We now can state our first main results.

Theorem 1.7. Let $(A, B)$ be a STA with a $B$-chain $\mathcal{C}: B=C_{0} \supset C_{1} \supset \cdots \supset C_{n-1} \supset$ $C_{n}=\{1\}$. Then $\mathcal{C}$ is a thin chain if and only if for all $i$ with $1 \leqslant i \leqslant n$ and all $b \in C_{i-1} \backslash C_{i}$,

$$
\delta(b)=o\left(C_{i}\right) / m(\mathcal{C}, i, b)
$$

ThEOREM 1.8. Let $(A, B)$ be a STA with a BT-chain $\mathcal{C}: B=C_{0} \supset C_{1} \supset \cdots \supset C_{n-1} \supset$ $C_{n}=\{1\}$. Let $b \in C_{i}$ for $i<n$. Then the following hold:

(i) $o\left(C_{i+1}\right) / \delta(b)$ is an integer divisor of o $\left(C_{i+1}\right)^{2}$. In particular, $o\left(O^{\vartheta}(B)\right) / \delta(b)$ is an integer divisor of $o\left(O^{\vartheta}(B)\right)^{2}$ for all $b \in B$. 
(ii) If each $C_{i}$ is normal in $B$ (for example, if $\mathcal{C}$ is a BTC-chain), then $o\left(C_{i+1}\right) / \delta(b)$ is an integer divisor of $o\left(C_{i+1}\right)$; hence, $\delta(b)$ is its complementary integer divisor.

Theorem 1.7 is proved in Section 3 below, and Theorem 1.8 in Section 4.

REMARK 1.9. A fusion ring $(A, B)$ is a table algebra with integer structure constants, and where $\lambda_{b b^{*} 1}=1$ for all $b \in B$. If $\phi(b)$ is the degree of such $b$, then $\phi(b)^{2}$ is the degree of the corresponding basis element in the rescaled standard basis. So Gelaki and Nikshych's result [8, Corollary 5.3] that $o\left(O^{\vartheta}(B)\right) / \phi(b)^{2}$ is an integer follows immediately.

Definition 1.10. Let $(A, B)$ be a STA. A TC-chain $\left\{Q_{i}\right\}_{i=0}^{q}$ is called terminal if $B=Q_{0} \supset Q_{1} \supset \cdots \supset Q_{q-1} \supset Q_{q}$, and there is no closed subset $Q_{q+1} \subset Q_{q}$ such that $\left\{Q_{i}\right\}_{i=0}^{q+1}$ is a TC-chain.

Definition 1.11. Let $(A, B)$ be a STA.

(i) The residual thin chain $\mathcal{R}$ is the chain $R_{0}=B, R_{1}=O^{\vartheta}(B), R_{i}=$ $O^{\vartheta}\left(R_{i-1}\right)$ for $1 \leqslant i$. Thus, $\mathcal{R}$ has length $r$, where $r$ is the least nonnegative integer with $R_{r+1}=R_{r}$.

(ii) The radical thin chain $\mathcal{J}$ is the chain $J_{0}=\{1\}, J_{1}=O_{\vartheta}(B), J_{i} / / J_{i-1}=$ $O_{\vartheta}\left(B / / J_{i-1}\right)$ for $1 \leqslant i$. Thus, $\mathcal{J}$ has length $j$, the least nonnegative integer with $J_{j+1}=J_{j}$.

(iii) The upper thin-central chain $\mathcal{Z}$ is the chain $Z_{0}=\{1\}, Z_{1}=O_{\vartheta}(B) \cap$ $Z(B), Z_{i} / / Z_{i-1}=O_{\vartheta}\left(B / / Z_{i-1}\right) \cap Z\left(B / / Z_{i-1}\right)$ for $1 \leqslant i$. Then $\mathcal{Z}$ has length $u$, the least nonnegative integer with $Z_{u+1}=Z_{u}$.

(iv) $A$ lower thin-central chain of length $q$ is a terminal $T C$-chain $\mathcal{Q}: Q_{0} \supset Q_{1} \supset$ $\ldots \supset Q_{q}$ such that $o\left(Q_{q}\right)$ is minimal, and $\sum_{i=0}^{q} o\left(Q_{i}\right)$ is minimal over all such chains. In particular, a lower BTC-chain of length $q$ is a BTC-chain $B=Q_{0} \supset Q_{1} \supset \cdots \supset Q_{q}=\{1\}$ such that $\sum_{i=0}^{q} o\left(Q_{i}\right)$ is minimal.

Definition 1.12. Let $(A, B)$ be a STA. Define $O^{\alpha}(B)$, the thin abelian residue, to be the unique closed subset of $B$ with $B \supseteq O^{\alpha}(B) \supseteq O^{\vartheta}(B)$ and $O^{\alpha}(B) / / O^{\vartheta}(B)$ is the commutator subgroup of the group $B / / O^{\vartheta}(B)$, that is, $\left[B / / O^{\vartheta}(B), B / / O^{\vartheta}(B)\right]$.

REMARK 1.13.

(i) Proposition 2.5(ii) below shows that $O^{\alpha}(B)$ is well-defined. From its definition and elementary group theory, it is the unique smallest closed subset of $B$ such that the quotient is an abelian group.

(ii) If $(A, B)$ is the group algebra of a finite group $G$, then $\left\{G_{i}\right\}_{i=0}^{q}$, where $G_{0}=$ $G, G_{1}=[G, G], G_{i}=\left[G, G_{i-1}\right]$ for all $1 \leqslant i \leqslant q$, where $G_{q+1}=G_{q}$, is the unique lower TC-chain for $(A, B)$.

(iii) Lower TC-chains for an arbitrary STA $(A, B)$ always exist, by the definition. If $(A, B)$ is nilpotent, then every lower BTC-chain of minimal length begins $B=Q_{0} \supset Q_{1}=O^{\alpha}(B)$, but is not necessarily unique. See Example 5.5 and Theorem 1.23 below. The example yields a negative answer to a question of Hanaki [9, Question 2.11] for association schemes.

The following theorem is proved by French and Zieschang [7, Theorem 6.1] in their more general context of hypergroups. We include a short proof in Section 4, for completeness.

Theorem 1.14. Let $(A, B)$ be a $S T A$. If $\mathcal{C}=\left\{C_{i}\right\}_{i=0}^{n}$ is a T-chain, $B=C_{0} \supset C_{1} \supset$ $\cdots \supset C_{n}$, then $C_{i} \supseteq R_{i}$ for all $0 \leqslant i \leqslant n$. Hence, $(A, B)$ is residually thin if and only if the residual $T$-chain $\mathcal{R}$ is bi-anchored. Furthermore, if $(A, B)$ is residually thin, and if $\mathcal{C}$ is any BT-chain of length $n$, then $n \geqslant r(r=$ the length of $\mathcal{R})$. 
DeFinition 1.15. The residual depth of a residually thin STA $(A, B)$ is the minimum length of all BT-chains in B; by Theorem 1.14, it equals $r$, the length of $\mathcal{R}$.

REMARK 1.16. The radical T-chain $\mathcal{J}$ in a residually thin STA of depth $r$ may be bi-anchored of arbitrarily large length $j>r$, or it may not be bi-anchored at all. See Example 4.2 below.

Definition 1.17. [5, Definition 1.4] Let $p$ be a prime. A STA $(A, B)$ is called pstandard if $o(B)=p^{N}$ for some integer $N>0$, and for all $b \in B, \delta(b)=p^{n_{b}}$ for some integer $n_{b} \geqslant 0$. An association scheme is called a $p$-scheme if its adjacency algebra is p-standard.

Proposition 1.18. Let $(A, B)$ be a STA with $o(B)=p^{N}$ for some prime $p$ and integer $N>0$. Then $(A, B)$ is p-standard if and only if it is residually thin.

REMARK 1.19. Not every $p$-standard table algebra is nilpotent. The example from [5, Remark 1.4], which is taken from [10, No.10851], is 2-standard of order 32, but has no nontrivial thin central basis elements.

We have the following analog of Theorem 1.14 for thin-central chains and nilpotent STAs. It is a straightforward generalization of [9, Theorem 2.5].

Theorem 1.20. Let $(A, B)$ be a STA. If $\mathcal{C}=\left\{C_{i}\right\}_{i=0}^{n},\{1\}=C_{0} \subset C_{1} \subset \cdots \subset C_{n}$ is a TC-chain, then $C_{i} \subseteq Z_{i}$ for all $0 \leqslant i \leqslant n$. Hence, $(A, B)$ is nilpotent if and only if the upper TC-chain $\mathcal{Z}$ is bi-anchored. Furthermore, if $(A, B)$ is nilpotent, and if $\mathcal{C}$ is any BTC-chain of length $n$, then $n \geqslant u(u=$ the length of $\mathcal{Z})$.

DEFINITION 1.21. The nilpotence class of a nilpotent $S T A(A, B)$ is the minimum length of all the BTC-chains in B. By Theorem 1.20, it equals u, the length of $\mathcal{Z}$.

It follows from Theorem 1.14 that for any nilpotent STA, the nilpotence class is at least the residual depth. If $A$ is commutative, then every T-chain is a TC-chain, hence by Theorem 1.20 the residual depth is at least the nilpotence class. So the following result of Gelaki and Nikshych is immediate.

Corollary 1.22. [8, Theorem 4.16] Let $(A, B)$ be a commutative STA. Then $(A, B)$ is residually thin iff $\mathcal{R}$ is bi-anchored iff $\mathcal{Z}$ is bi-anchored iff $(A, B)$ is nilpotent; and in this case, the residual depth and nilpotence class of $(A, B)$ are equal.

THEOREM 1.23. If $(A, B)$ is a nilpotent $S T A$, then every lower BTC-chain of length $u$ begins $B \supset O^{\alpha}(B)$.

Preliminary results (mostly known) and some further definitions are collected in Section 2. Theorem 1.7 and a related more general result are proved in Section 3. Section 4 contains proofs of Theorem 1.14, Theorem 1.8, Proposition 1.18, and other structural results for residually thin STAs. In particular, we show that if $D$ is any closed subset of a residually thin table basis $B$, then $o(D)$ is an integer such that $o(D) \mid o(B)$. Example 4.2 is also presented. Section 5 establishes Theorem 1.20 and Theorem 1.23, and studies further aspects of TC-chains and nilpotent STAs. In particular, Corollary 5.4 shows that a STA $(A, B)$ is nilpotent if and only if $B / / O^{\vartheta}(B)$ is nilpotent as a group, and $Z_{k} \supseteq O^{\vartheta}(B)$ for some integer $k$. Example 5.5 demonstrates that lower BTC-chains in a nilpotent STA are not always unique.

\section{Preliminaries}

The results in the section for which proofs are omitted are known; proofs for them are given in [1], [6], or [3]. Throughout, $(A, B)$ is a table algebra (TA).

There is a positive definite Hermitian form ( , ) on $A$ such that for all $b, c, d \in B$, 


$$
(b, c)=\beta_{b c^{*} 1} ; \text { and }(b c, d)=\left(b, d c^{*}\right)=\left(c, b^{*} d\right) .
$$

Definition 2.1. Let $(A, B),(U, V)$ be TAs. $A$ table algebra (TA-)homomorphism $\psi$ : $(A, B) \rightarrow(U, V)$ is an algebra homomorphism $\psi: A \rightarrow U,\left(\psi\left(1_{A}\right)=1_{U}\right)$, such that for each $b \in B$, there is some $v \in V$ and $\alpha_{b} \in \mathbb{R}_{>0}$ with $\psi(b)=\alpha_{b} v$. Define $V_{\psi}:=$ $\left\{v \in V \mid \psi(b)=\alpha_{b} v\right.$ for some $b \in B$ and $\left.\alpha_{b} \in \mathbb{R}_{>0}\right\}$. Then $V_{\psi}$ is a closed subset of $V$. Now $\psi$ is called a TA-isomorphism if $\psi$ is one-to-one and $V_{\psi}=V$. This is equivalent to $\psi: A \rightarrow U$ being an algebra isomorphism. If there exists such a TA-isomorphism, we write $(A, B) \cong(U, V)$.

Definition 2.2. The kernel of a TA-homomorphism $\psi$ is defined as $\operatorname{ker} \psi:=\{b \in$ $B \mid \psi(b)=\alpha_{b} 1_{U}$ for some $\left.\alpha_{b} \in \mathbb{R}_{>0}\right\}$.

Then $\psi$ preserves the respective anti-automorphisms, ker $\psi$ is a normal closed subset of $B$, and the following "Fundamental Homomorphism Theorem" holds:

Proposition 2.3. Let $\psi:(A, B) \rightarrow(U, V)$ be a TA-epimorphism of STAs. Let $C=\operatorname{ker} \psi$. Let $e=e_{C}:=o(C)^{-1} C^{+}$, a central idempotent of $A$. Then $\pi:(A, B) \rightarrow$ $(A / / C, B / / C)$, where $\pi(b)=$ be for all $b \in B$, is a TA-epimorphism, as be $=$ $(\delta(b) / \delta(b / / C)) b / / C$. Furthermore, there is a TA-isomorphism $\bar{\psi}:(A / / C, B / / C) \rightarrow$ $(U, V)$ such that $\bar{\psi} \circ \pi=\psi$. Finally, since both $(A / / C, B / / C)$ and $(U, V)$ are standard, then for all $b \in B, \bar{\psi}(b / / C)=v$, where $\psi(b)=\alpha_{b} v$ for some $\alpha_{b} \in \mathbb{R}_{>0}$.

Proposition 2.3 has the following consequence:

Lemma 2.4. Let $(A, B)$ be a STA with closed subsets $C, D$ where $D$ is normal in $B$. Then $D C=C D$ is a closed subset, $D \cap C$ is a normal closed subset of $C$, and

$$
C / /(D \cap C) \cong C D / / D,
$$

via a TA-isomorphism that yields the correspondence $c / / D \cap C \leftrightarrow c / / D$, for all $c \in C$.

The first two parts of the next proposition are contained in [6, Proposition 2.13], and part (iii) is proved for hypergroups in [7, Lemma 4.6]. Parts (iii), (iv), and (v) are proved below.

Proposition 2.5. Let $(A, B)$ be a STA and $C$ a closed subset of $B$. Then the following hold:

(i) The correspondence $D \mapsto D / / C$ is a bijection between the set of closed subsets of $B$ that contain $C$ and the set of closed subsets of $B / / C$.

(ii) Suppose that $D$ is a closed subset of $B$ with $C \subseteq D \subseteq B$. Then for all $b \in B$, $(b / / C) / /(D / / C)=b / / D$. Hence, $(B / / C) / /(D / / C)=B / / D$.

(iii) Suppose that $C \subseteq D \subseteq B$. Then $D$ is strongly normal in $B$ if and only if $D / / C$ is strongly normal in $B / / C$.

(iv) Suppose that $C \subseteq D \subseteq B$ and $D$ is normal in $B$. Then $D / / C$ is normal in $B / / C$.

(v) Suppose that $C \subseteq D \subseteq B$ and $C$ is normal in $B$. Then $D$ is a normal subset of $B$ if and only if $D / / C$ is a normal subset of $B / / C$.

Proof. (iii) $D$ is strongly normal in $B$ iff $B / / D$ is thin iff $(B / / C) / /(D / / C)$ is thin (by (ii)) iff $D / / C$ is strongly normal in $B / / C$.

(iv) Since $D$ is assumed normal in $B,(C b C) D=D(C b C)$ for all $b \in B$. It follows that $b / / C \cdot D / / C=D / / C \cdot b / / C$, hence $D / / C$ is a normal subset of $B / / C$.

(v) Suppose that $C$ is a normal subset of $B$, and that $D / / C$ is normal in $B / / C$. Then for all $b \in B, b / / C \cdot D / / C=D / / C \cdot b / / C$, hence $(C b C) D=D(C b C)$. But $C \subseteq D$, so $C D=D=D C$. Thus we have $C b D=D b C$. Since $C$ is normal in $B$, $C b D=b C D=b D$, and $D b C=D C b=D b$. The result follows. 
Lemma 2.6. Let $(A, B)$ be a STA with closed subsets $C_{1} \supseteq C_{2}$ and $D$ such that $C_{2}$ is strongly normal in $C_{1}$, and for all $c \in C_{1}, c D \subseteq D c C_{2}$. Then $D C_{1}$ and $D C_{2}$ are closed subsets such that $D C_{2}$ is strongly normal in $D C_{1}$.

Proof. It follows from the hypothesis that $C_{1} D \subseteq D C_{1}$. Then $C_{1} D=\left(D C_{1}\right)^{*} \supseteq$ $\left(C_{1} D\right)^{*}=D C_{1}$. So $D C_{1}=C_{1} D$, hence $D C_{1}$ is a closed subset. The hypothesis restricted to $c \in C_{2}$ similarly yields $D C_{2}=C_{2} D$, hence $D C_{2}$ is closed. Any $b \in D C_{1}$ is in $\operatorname{Supp}(d c)$ for some $d \in D, c \in C_{1}$. Then set products

$$
b D C_{2} b^{*} \subseteq d c D C_{2} c^{*} d^{*}=d(c D) C_{2} c^{*} d^{*} \subseteq d\left(D c C_{2}\right) C_{2} c^{*} d^{*}=D\left(c C_{2} c^{*}\right) d^{*} .
$$

Now $C_{2}$ strongly normal in $C_{1}$ and $C_{2} D=D C_{2}$ yield

$$
D\left(c C_{2} c^{*}\right) d^{*}=D C_{2} d^{*}=D\left(C_{2} d^{*}\right) \subseteq D\left(D C_{2}\right)=D C_{2} .
$$

Remark 2.7. Given closed subsets $C_{1} \supseteq C_{2}$ and $D$ with $C_{2}$ strongly normal in $C_{1}$, the final hypothesis of Lemma 2.6 will follow from either $D$ normal in $B$, or both $C_{1}$ and $C_{2}$ normal in $B$ with $\left(c / / C_{2}\right) \cdot\left(D C_{2} / / C_{2}\right)=\left(D C_{2} / / C_{2}\right) \cdot\left(c / / C_{2}\right)$ for all $c \in C_{1}$. This is because the latter assumption implies that $c D C_{2}=\left(C_{2} c C_{2}\right)\left(D C_{2}\right)=$ $\left(D C_{2}\right)\left(C_{2} c C_{2}\right)=D c C_{2}$.

Lemma 2.8. Let $(A, B)$ be a $S T A$ with closed subset $C$ and normal closed subset $D$. If $O^{\vartheta}(C) \subseteq D$, then $C / /(D \cap C)$ is thin.

Proof. Lemma 2.6 implies that $D O^{\vartheta}(C)$ is strongly normal in $D C$. But $O^{\vartheta}(C) \subseteq D$ by hypothesis, so $D$ is strongly normal in $D C$. Hence, $D C / / D$ is thin. By Lemma 2.4, $C / /(D \cap C)$ is thin.

Lemma 2.9. Let $(A, B)$ be a STA, $C$ a closed subset of $B$, and $b \in B$. Then $\delta$ is constant over the double coset $C b C$ if and only if o $(C b C)=\operatorname{card}(C b C) \delta\left(b^{\prime}\right)$ for all $b^{\prime} \in C b C$.

Proof. Since $o(C b C)=\sum_{b^{\prime} \in C b C} \delta\left(b^{\prime}\right)$ and $\operatorname{card}(C b C)$ are independent of any particular $b^{\prime} \in C b C$, the proof is immediate.

Lemma 2.10. Let $(A, B)$ be a STA, $C$ a thin closed subset of $B$, and $b \in B$. Then $\operatorname{card}(C b C) \mid o(C)^{2}$. If $C$ is normal in $B$, then $\operatorname{card}(C b C) \mid o(C)$.

Proof. The group $C \times C$ acts on the double coset $C b C$ as follows: for all $c_{1}, c_{2}, x, y, \in C$,

$$
\left(c_{1} b c_{2}\right)^{(x, y)}=x^{-1} c_{1} b c_{2} y .
$$

The double coset itself is the sole orbit under this action. Hence, if $S:=\{(x, y) \in C \times$ $\left.C \mid x^{-1} b y=b\right\}$, i.e. $S$ is the stabilizer of $b$ under the action, then $\operatorname{card}(C b C)=\mid C \times C$ : $S \mid$. If $C$ is normal in $B$, then $C b C=b C$, and $C$ acts on $b C$ by right multiplication. If $S^{r}$ denotes the stabilizer of $b$ in $C$ under this action, then $\operatorname{card}(C b C)=\left|C: S^{r}\right|$.

LEMmA 2.11. Let $(A, B)$ be a $S T A$, and $\mathcal{C}=\left\{C_{i}\right\}_{i=0}^{n}$ a BT-chain with $B=C_{0} \supset C_{1} \supset$ $\cdots \supset C_{n}=\{1\}$. Then for all $1 \leqslant i \leqslant n$ and all $b \in B \backslash C_{i}, m(\mathcal{C}, i, b) \mid o\left(C_{i}\right)^{2}$. If each $C_{i}$ is normal in $B$ (in particular, if $\mathcal{C}$ is a BTC-chain), then $m(\mathcal{C}, i, b) \mid o\left(C_{i}\right)$.

Proof. This is immediate from the definitions if $i=n$, so assume $1 \leqslant i<n$ and $b \in B \backslash C_{i}$. Lemma 2.10 implies that for each $j$ with $i \leqslant j \leqslant n-1$,

$$
\operatorname{card}\left(C_{j} / / C_{j+1} \cdot b / / C_{j+1} \cdot C_{j} / / C_{j+1}\right) \mid o\left(C_{j} / / C_{j+1}\right)^{2}=o\left(C_{j}\right)^{2} / o\left(C_{j+1}\right)^{2} .
$$

Hence,

$$
m(\mathcal{C}, i, b)=\prod_{j=i}^{n-1} \operatorname{card}\left(C_{j} / / C_{j+1} \cdot b / / C_{j+1} \cdot C_{j} / / C_{j+1}\right) \mid \prod_{j=i}^{n-1} \frac{o\left(C_{j}\right)^{2}}{o\left(C_{j+1}\right)^{2}}=o\left(C_{i}\right)^{2} .
$$


If each $C_{i}$ is normal in $B$, then each $C_{j} / / C_{j+1}$ is a thin normal closed subset of $B / / C_{j+1}$, by Proposition 2.5(iv). So Lemma 2.10 yields that for all $i \leqslant j \leqslant n-1$, $\operatorname{card}\left(C_{j} / / C_{j+1} \cdot b / / C_{j+1} \cdot C_{j} / / C_{j+1}\right)=\operatorname{card}\left(b / / C_{j+1} \cdot C_{j} / / C_{j+1}\right) \mid o\left(C_{j} / / C_{j+1}\right)$.

Therefore, $m(\mathcal{C}, i, b) \mid \prod_{j=i}^{n-1}\left(o\left(C_{j}\right) / o\left(C_{j+1}\right)\right)=o\left(C_{i}\right)$.

\section{Degrees And Double Cosets}

Throughout this section, $(A, B)$ is a STA with degree map $\delta$, and $\mathcal{C}=\left\{C_{i}\right\}_{i=0}^{n}$ is a B-chain with $B=C_{0} \supset C_{1} \cdots \supset C_{n}=\{1\}$. We prove a theorem that, for a given B-chain, yields a criterion whereby the degree of a quotient element is found in terms of the cardinality of double cosets in quotient bases determined by the chain. The result is applied to prove Theorem 1.7.

Definition 3.1. Fix integer $k$ with $0<k<n$. Define the chain

$$
\mathcal{C} / / C_{k}:=\left\{C_{i} / / C_{k}\right\}_{i=0}^{k} .
$$

Remark 3.2. We have by Proposition 2.5(i) that

$$
B / / C_{k}=C_{0} / / C_{k} \supset C_{1} / / C_{k} \cdots \supset C_{k-1} / / C_{k} \supset C_{k} / / C_{k},
$$

so that $\mathcal{C} / / C_{k}$ is indeed a B-chain of length $k$ in $B / / C_{k}$.

Lemma 3.3. Fix integers $i, k$ with $0 \leqslant i \leqslant k \leqslant n$, so that $C_{i} \supseteq C_{k}$. Then for all $b \in B \backslash C_{i}$,

$$
m(\mathcal{C}, i, b)=m\left(\mathcal{C} / / C_{k}, i, b / / C_{k}\right) \cdot m(\mathcal{C}, k, b) .
$$

Proof. If $i=k$, then $m\left(\mathcal{C} / / C_{k}, k, b / / C_{k}\right)=1$ by definition, as $\mathcal{C} / / C_{k}$ has length $k$. If $k=n$, then $m(\mathcal{C}, n, b)=1, \mathcal{C} / / C_{n}=\mathcal{C}$, and $b / / C_{n}=b$. So we may assume that $i<k<n$. Proposition 2.5(ii) implies for all $i \leqslant j \leqslant k-1$,

$$
\begin{gathered}
\left(\left(C_{j} / / C_{k}\right) / /\left(C_{j+1} / / C_{k}\right)\right) \cdot\left(\left(b / / C_{k}\right) / /\left(C_{j+1} / / C_{k}\right)\right) \cdot\left(\left(C_{j} / / C_{k}\right) / /\left(C_{j+1} / / C_{k}\right)\right) \\
=C_{j} / / C_{j+1} \cdot b / / C_{j+1} \cdot C_{j} / / C_{j+1},
\end{gathered}
$$

so that by Definition 1.6, $m\left(\mathcal{C} / / C_{k}, i, b / / C_{k}\right)=\prod_{i \leqslant j \leqslant k-1} \operatorname{card}\left(C_{j} / / C_{j+1} \cdot b / / C_{j+1}\right.$. $\left.C_{j} / / C_{j+1}\right)$. Since by the same definition $m(\mathcal{C}, k, b)$ (resp. $\left.m(\mathcal{C}, i, b)\right)$ equals the analogous product over $k \leqslant j \leqslant n-1$ (resp. $i \leqslant j \leqslant n-1$ ), the result follows.

Theorem 3.4. Let $\mathcal{C}=\left\{C_{i}\right\}_{i=0}^{n}$ be a B-chain with $B=C_{0} \supset C_{1} \supset \cdots \supset C_{n}=\{1\}$. Then $\delta$ is constant on each double coset $C_{j} / / C_{j+1} \cdot b / / C_{j+1} \cdot C_{j} / / C_{j+1}$ for all $0 \leqslant$ $i \leqslant j \leqslant n-1$ and all $b \in B \backslash C_{i}$ if and only if, for all $0 \leqslant i \leqslant n-1$,

$$
\delta\left(b / / C_{i}\right)=\frac{\delta(b)}{o\left(C_{i}\right)} m(\mathcal{C}, i, b) .
$$

Proof. Fix $i \geqslant 0$. By Proposition 2.5(ii), $\delta$ is constant on $C_{j} / / C_{j+1} \cdot b / / C_{j+1} \cdot C_{j} / / C_{j+1}$ for all $i \leqslant j \leqslant n-1$ and all $b \in B \backslash C_{i}$ if and only if $\delta$ is constant on

(1) $\left(\left(C_{j} / / C_{n-1}\right) / /\left(C_{j+1} / / C_{n-1}\right)\right) \cdot\left(\left(b / / C_{n-1}\right) / /\left(C_{j+1} / / C_{n-1}\right)\right)$

$\cdot\left(\left(C_{j} / / C_{n-1}\right) / /\left(C_{j+1} / / C_{n-1}\right)\right)$ for all $i \leqslant j \leqslant n-2$ and all $b \in B \backslash C_{i}$,

and

$$
\delta \text { is constant on } C_{n-1} b C_{n-1} \text { for all } b \in B \backslash C_{i} .
$$

Since $B \backslash C_{i}$ is a union of $C_{n-1}-C_{n-1}$ double cosets, Lemma 2.9 implies that (2) is equivalent to $\delta\left(b / / C_{n-1}\right)=\frac{\delta(b)}{o\left(C_{n-1}\right)} m(\mathcal{C}, n-1, b)$ for all $b \in B \backslash C_{i}$. So if $i=n-1$, the theorem is proved. 
Suppose that $i<n-1$. By induction on $n$, (1) is equivalent to

$$
\delta\left(\left(b / / C_{n-1}\right) / /\left(C_{i} / / C_{n-1}\right)\right)=\frac{\delta\left(b / / C_{n-1}\right)}{o\left(C_{i} / / C_{n-1}\right)} m\left(\mathcal{C} / / C_{n-1}, i, b / / C_{n-1}\right)
$$

for all $b \in B \backslash C_{i}$. So if (1) and (2) both hold, we have

$$
\begin{aligned}
\delta\left(b / / C_{i}\right) & =\delta\left(\left(b / / C_{n-1}\right) / /\left(C_{i} / / C_{n-1}\right)\right)=\frac{\delta\left(b / / C_{n-1}\right)}{o\left(C_{i}\right) / o\left(C_{n-1}\right)} m\left(\mathcal{C} / / C_{n-1}, i, b / / C_{n-1}\right) \\
& =\frac{\delta(b)}{o\left(C_{n-1}\right) o\left(C_{i}\right) / o\left(C_{n-1}\right)} m(\mathcal{C}, n-1, b) \cdot m\left(\mathcal{C} / / C_{n-1}, i, b / / C_{n-1}\right) .
\end{aligned}
$$

Hence by Lemma $3.3, \delta\left(b / / C_{i}\right)=\frac{\delta(b)}{o\left(C_{i}\right)} m(\mathcal{C}, i, b)$.

Conversely, suppose that for all $0 \leqslant i \leqslant n$ and all $b \in B \backslash C_{i}, \delta\left(b / / C_{i}\right)=$ $\frac{\delta(b)}{o\left(C_{i}\right)} m(\mathcal{C}, i, b)$. If $i=n-1$, we have already shown that $\delta$ is constant on $C_{n-1} b C_{n-1}$. Suppose that $i<n-1$. Then by Lemma 3.3,

$$
\begin{aligned}
\delta\left(b / / C_{i}\right) & =\frac{\delta(b)}{o\left(C_{i}\right)} m(\mathcal{C}, n-1, b) \cdot m\left(\mathcal{C} / / C_{n-1}, i, b / / C_{n-1}\right) \\
& =\frac{o\left(C_{n-1}\right)}{o\left(C_{i}\right)} \delta\left(b / / C_{n-1}\right) m\left(\mathcal{C} / / C_{n-1}, i, b / / C_{n-1}\right) .
\end{aligned}
$$

So

$$
\delta\left(\left(b / / C_{n-1}\right) / /\left(C_{i} / / C_{n-1}\right)\right)=\frac{\delta\left(b / / C_{n-1}\right)}{o\left(C_{i} / / C_{n-1}\right)} m\left(\mathcal{C} / / C_{n-1}, i, b / / C_{n-1}\right)
$$

for all $0 \leqslant i \leqslant n-2$ and $b \in B \backslash C_{i}$, whence $\mathcal{C} / / C_{n-1}$ satisfies the same hypothesis as $\mathcal{C}$. Then by induction on $n, \delta$ is constant on all double cosets

$$
\begin{aligned}
&\left(\left(C_{j} / / C_{n-1}\right) / /\left(C_{j+1} / / C_{n-1}\right)\right) \cdot\left(\left(b / / C_{n-1}\right) / /\left(C_{j+1} / / C_{n-1}\right)\right) \\
& \cdot\left(\left(C_{j} / / C_{n-1}\right) / /\left(C_{j+1} / / C_{n-1}\right)\right)=C_{j} / / C_{j+1} \cdot b / / C_{j+1} \cdot C_{j} / / C_{j+1},
\end{aligned}
$$

for all $i \leqslant j \leqslant n-2$ and $b \in B \backslash C_{i}$. This establishes the converse.

Proof of Theorem 1.7. Observe that $C_{n-1}$ is thin iff $\delta(b)=1$ for all $b \in C_{n-1} \backslash C_{n}$ iff $\delta(b)=o\left(C_{n}\right) / m(\mathcal{C}, n, b)$.

If $\mathcal{C}$ is thin, then $C_{j} / / C_{j+1}$ is a thin closed subset for all $0 \leqslant j \leqslant n-1$, by definition. So $\delta$ is constant on each double coset $C_{j} / / C_{j+1} \cdot b / / C_{j+1} \cdot C_{j} / / C_{j+1}$ for all $0 \leqslant i \leqslant j \leqslant n-1$ and all $b \in B \backslash C_{i}$. Hence, $\delta\left(b / / C_{i}\right)=\frac{\delta(b)}{o\left(C_{i}\right)} m(\mathcal{C}, i, b)$ for all $0 \leqslant i \leqslant n-1$ and $b \in B \backslash C_{i}$, by Theorem 3.4. But $C_{i-1} / / C_{i}$ thin and $b \in C_{i-1} \backslash C_{i}$ imply that $\delta\left(b / / C_{i}\right)=1$. Hence, $\delta(b)=o\left(C_{i}\right) / m(\mathcal{C}, i, b)$ for $0 \leqslant i \leqslant n-1$.

Conversely, suppose that $\delta(b)=o\left(C_{i}\right) / m(\mathcal{C}, i, b)$ for all $0 \leqslant i \leqslant n$ and all $b \in$ $C_{i-1} \backslash C_{i}$. In particular if $i=n$, then $C_{n-1}$ is thin, as noted above. So $\delta$ is constant on $C_{n-1} b C_{n-1}$ for all $b \in B$.

If $i<n$ and $b \in C_{i-1} \backslash C_{i}$, then $C_{n-1}$ thin, Lemma 2.9 and Lemma 3.3 imply that

$\delta\left(b / / C_{n-1}\right)=\frac{\delta(b)}{o\left(C_{n-1}\right)} m(\mathcal{C}, n-1, b)=\frac{o\left(C_{i}\right) m(\mathcal{C}, n-1, b)}{m(\mathcal{C}, i, b) o\left(C_{n-1}\right)}=\frac{o\left(C_{i} / / C_{n-1}\right)}{m\left(\mathcal{C} / / C_{n-1}, i, b / / C_{n-1}\right)}$.

So the same hypothesis holds for the chain $\mathcal{C} / / C_{n-1}$ in $B / / C_{n-1}$ as for $\mathcal{C}$. Induction on $n$ implies that $\mathcal{C} / / C_{n-1}$ is thin. Since $C_{j} / / C_{j+1}=\left(C_{j} / / C_{n-1}\right) / /\left(C_{j+1} / / C_{n-1}\right)$ for all $0 \leqslant j \leqslant n-2$ by Proposition 2.5 (ii), $\mathcal{C}$ is thin. 


\section{Residually thin STAs}

In this section, we prove Theorem 1.14, Theorem 1.8, Proposition 1.18, and in Theorem 4.1 properties of residually thin STAs. Parts of the latter result are proved for hypergroups in [7], as noted below; but we include proofs here, as they fit easily into our context. Example 4.2 below illustrates how much residual depth and radical length can differ. Throughout, $(A, B)$ denotes a STA.

Proof of Theorem 1.14. Let $\mathcal{C}=\left\{C_{i}\right\}_{i=0}^{n}$ be a T-chain in $B, B=C_{0} \supset C_{1} \supset$ $\cdots \supset C_{n-1} \supset C_{n}$. Then $B / / C_{1}$ is thin, by Definition 1.2. Since $R_{1}=O^{\vartheta}(B)$ is the unique minimal closed subset of $B$ such that $B / / R_{1}$ is thin, $C_{1} \supseteq R_{1}$. Suppose that $C_{i} \supseteq R_{i}$ for some $i \geqslant 1$. Since by definition $C_{i} / / C_{i+1}$ is thin, $C_{i+1}$ is (strongly) normal in $C_{i}$. Hence, $R_{i} C_{i+1}=C_{i+1} R_{i}$ is a closed subset of $C_{i}$. So by Proposition 2.5(i), $R_{i} C_{i+1} / / C_{i+1}$ is a subgroup of $C_{i} / / C_{i+1}$. By Lemma 2.4 , $R_{i} / /\left(R_{i} \cap C_{i+1}\right) \cong R_{i} C_{i+1} / / C_{i+1}$. Thus, $R_{i} / /\left(R_{i} \cap C_{i+1}\right)$ is thin. Then by the definition of $\mathcal{R}, R_{i+1} \subseteq R_{i} \cap C_{i+1} \subseteq C_{i+1}$. It follows by induction that $C_{i} \supseteq R_{i}$ for $0 \leqslant i \leqslant n$. So if $C_{n}=\{1\}$, then $R_{n}=\{1\}$ and $n \geqslant r$. The theorem follows.

Proof of Theorem 1.8. Let $b \in C_{i}$. We may assume that $b \neq 1$. So for some $j$ with $i \leqslant j<n, b \in C_{j} \backslash C_{j+1}$. Theorem 1.7 then implies that $o\left(C_{j+1}\right) / \delta(b)=m(\mathcal{C}, j+1, b)$, which is an integer. Lemma 2.11 yields further that $o\left(C_{j+1}\right) / \delta(b) \mid o\left(C_{j+1}\right)^{2}$. As $j+1 \leqslant i+1, o\left(C_{j+1}\right) \mid o\left(C_{i+1}\right)$ by Proposition 1.5. Hence, $o\left(C_{i+1}\right) / \delta(b)$ is an integer and $o\left(C_{i+1}\right) / \delta(b) \mid o\left(C_{i+1}\right)^{2}$. If each $C_{j}$ is normal in $B$, then Lemma 2.11 implies that $o\left(C_{j+1}\right) / \delta(b) \mid o\left(C_{j+1}\right)$, so $o\left(C_{i+1}\right) / \delta(b) \mid o\left(C_{i+1}\right)$.

By Theorem 1.14, the residual thin chain $\mathcal{R}$ is also bi-anchored. Since $b \in B=$ $R_{0}$, and $R_{1}=O^{\vartheta}(B)$, our proof shows that $o\left(O^{\vartheta}(B)\right) / \delta(b)$ is an integer divisor of $o\left(O^{\vartheta}(B)\right)^{2}$.

Proof of Proposition 1.18. Suppose that $(A, B)$ is $p$-standard. By [5, Proposition 3.2], there is a chain $\{1\} \subset C_{1} \subset C_{2} \subset \cdots \subset C_{N}=B$ with $C_{i+1} / / C_{i}$ a cyclic group of order $p$ for $0 \leqslant i<N$. So by Definition $1.3,(A, B)$ is residually thin. Suppose that $(A, B)$ is residually thin. Let $\left\{R_{i}\right\}_{i=0}^{r}$ be the residual thin chain, so that $B=R_{0} \supset R_{1} \supset$ $\cdots \supset R_{r}=\{1\}$, by Theorem 1.14. By Proposition 1.5,o $\left(R_{1}\right)$ is an integer divisor of $o(B)=p^{N}$. Thus, $o\left(R_{1}\right)=p^{N_{1}}$ for some nonnegative integer $N_{1}<N$. For each $b \in B, p^{N_{1}} / \delta(b)=o\left(R_{1}\right) / \delta(b)$ is an integer divisor of $o\left(R_{1}\right)^{2}=p^{2 N_{1}}$ by Theorem 1.8. It follows that $\delta(b)$ is a power of $p$, and $(A, B)$ is $p$-standard.

Part (i) and most of (ii) of the next result are proved for hypergroups by French and Zieschang in [7, Theorem 6.3]. Since the proofs in our context are shorter, they are included below. Part (iii) seems new.

THEOREM 4.1. Let $(A, B)$ be a residually thin STA of depth $n$, with a BT-chain $\left\{C_{i}\right\}_{i=0}^{n}, B=C_{0} \supset C_{1} \supset \cdots \supset C_{n}=\{1\}$. Let $D$ be any closed subset of $B$.

(i) The distinct members of $\left\{D \cap C_{i}\right\}_{i=0}^{n}$ form a BT-chain for $D$, hence $D$ is residually thin of depth at most $n$. In particular, $o(D)$ is an integer.

(ii) Suppose either that $D$ is normal in $B$, or that each $C_{i}$ is normal in $B$ and $\left(c / / C_{i+1}\right) \cdot\left(D C_{i+1} / / C_{i+1}\right)=\left(D C_{i+1} / / C_{i+1}\right) \cdot\left(c / / C_{i+1}\right)$ for all $c \in C_{i}$. Then the distinct members of $\left\{D C_{i} / / D\right\}_{i=0}^{n}$ form a BT-chain for $B / / D$, hence $B / / D$ is residually thin of depth at most $n$.

(iii) $o(D) \mid o(B)$.

Proof. Let $b \in D \cap C_{i}$. Then $b\left(D \cap C_{i+1}\right) b^{*} \subseteq C_{i+1}$, since $C_{i+1}$ is strongly normal in $C_{i}$; and $b\left(D \cap C_{i+1}\right) b^{*} \subseteq D$, since $b \in D$ and $D$ is closed. Thus, $b\left(D \cap C_{i+1}\right) b^{*} \subseteq D \cap C_{i+1}$. So $D \cap C_{i+1}$ is strongly normal in $D \cap C_{i}$. Hence, $\left(D \cap C_{i}\right) / /\left(D \cap C_{i+1}\right)$ is thin. So 
$D$ is residually thin of depth at most $n$, and $o(D)$ is an integer by Proposition 1.5. Thus, (i) follows.

Assume the hypotheses of (ii). Then each $D C_{i}$ is closed, and the hypotheses of Lemma 2.6 hold for each pair $C_{i}, C_{i+1}$, by Remark 2.7. By Lemma 2.6, each $D C_{i+1}$ is strongly normal in $D C_{i}$. So $D C_{i} / / D C_{i+1}=\left(D C_{i} / / D\right) / /\left(D C_{i+1} / / D\right)$ is thin. Therefore, the distinct members of $\left\{D C_{i} / / D\right\}_{i=0}^{n}$ form a BT-chain for $B / / D$, and (ii) is proved.

Now $C_{1}$ is residually thin of depth $n-1$, and is strongly normal in $B$; and $D \cap C_{1}$ is a closed subset of $C_{1}$. So $o\left(D \cap C_{1}\right) \mid o\left(C_{1}\right)$ by induction on $n$. Since $D C_{1} / / C_{1}$ is a subgroup of the group $B / / C_{1}$, we have $o\left(D C_{1} / / C_{1}\right) \mid o\left(B / / C_{1}\right)=o(B) / o\left(C_{1}\right)$. By Lemma $2.4, D / /\left(D \cap C_{1}\right) \cong D C_{1} / / C_{1}$. Thus,

$$
o(D) / o\left(D \cap C_{1}\right)=o\left(D / /\left(D \cap C_{1}\right)\right)=o\left(D C_{1} / / C_{1}\right) \mid o(B) / o\left(C_{1}\right) .
$$

Hence,

$$
o(D)\left|\frac{o(B) o\left(D \cap C_{1}\right)}{o\left(C_{1}\right)}=\frac{o(B)}{o\left(C_{1}\right) / o\left(D \cap C_{1}\right)}\right| o(B),
$$

where the last division holds because $o\left(C_{1}\right) \mid o(B)$ by Proposition 1.5, and $o\left(D \cap C_{1}\right) \mid$ $o\left(C_{1}\right)$. Therefore, (iii) holds.

EXAmple 4.2. Let $G$ be a finite group, $H$ a proper subgroup of $G, Y$ any other finite group, and $C=H \times Y$. Define the group homomorphism $\psi: C \rightarrow H$ by $\psi(h y)=h$ for all $h \in H, y \in Y$. Consider the partial wreath product $C \circ_{\psi} G=C \cup(G \backslash H)$ as a basis for a vector space over $\mathbb{C}$ [2, Definition 4.1, Definition 4.2], [4, Definition 1.13, Definition 2.4]. That is, $H$ is replaced inside $G$ by $C$. Multiplication in $\mathbb{C}\left(C \circ_{\psi} G\right)$ is defined as follows: for all $h \in H, y \in Y, g \in G \backslash H,(h y) \cdot g=h g$ (the product in $G)$, and $g \cdot(h y)=g h$. If $g_{1}, g_{2} \in G \backslash H$ with $g_{1} g_{2}=h \in H$, then in $\mathbb{C}\left(C \circ_{\psi} G\right)$, $g_{1} g_{2}=\frac{1}{o(Y)} h Y^{+}=\frac{1}{o(Y)} Y^{+} h$. The other products are as in $G$ or $C$. Rescale each $g \in G \backslash H$ as $b:=o(Y) g$. Define $B$ as the rescaled $C \circ_{\psi} G$ and $A:=\mathbb{C} B$. Then $(A, B)$ is a STA. For all $c \in C, c^{*}=c^{-1}$ and $c c^{*}=1$. For all $g \in G \backslash H$ and $b=o(Y) g$, $b^{*}=o(Y) g^{-1}$ and $b b^{*}=o(Y) Y^{+}$. Hence, $O_{\vartheta}(B)=C$, and $O^{\vartheta}(B)=Y$. Then the residual thin chain $\mathcal{R}$ is

$$
B \supset O^{\vartheta}(B)=Y \supset\{1\},
$$

and $(A, B)$ is residually thin of depth 2 .

Suppose that $H=N_{G}(H)$; that is, $H$ is its own normalizer in the group $G$. Then for all $b=o(Y) g \in B \backslash C$, the set product

$$
b C b^{*}=\left\{o(Y) g_{1} \mid g_{1} \in g H g^{-1} \backslash H\right\} \cup\left(\left(g H g^{-1} \cap H\right) \times Y\right) \not L H \times Y,
$$

since $g \notin N_{G}(H)$. So if $D$ is any closed subset of $B$ with $C \subset D \subseteq B$, then $C$ is not strongly normal in $D$, hence $D / / C$ is not thin. It follows that the radical thin chain $\mathcal{J}$ is $\{1\} \subset C$, of length 1 and not bi-anchored.

Let $n>1$ be an arbitrary integer, and suppose that $G$ is a group with a chain of subgroups $H=H_{1}<H_{2}<\cdots<H_{n}=G$, such that $N_{G}\left(H_{i}\right)=H_{i+1}$ for $1 \leqslant i \leqslant n-1$. (This is the case, for example, if $G=D_{2^{n}}$, the dihedral group of order $2^{n}, u$ is a noncentral involution in $G,\langle v\rangle$ is the cyclic subgroup of order $2^{n-1}$, and $H_{i}=\left\langle v^{2^{n-i}}\right\rangle \cup\left\langle v^{2^{n-i}}\right\rangle u$ for $1 \leqslant i \leqslant n$.) Let $C_{0}=\{1\}, C_{1}=C$, and $C_{i}=C \cup\left\{o(Y) g \mid g \in H_{i} \backslash H\right\}$ for $2 \leqslant i \leqslant n$. Then for each $1 \leqslant i \leqslant n-1, C_{i}$ is a closed subset that is strongly normal in $C_{i+1}$, but $b C_{i} b^{*} \nsubseteq C_{i}$ for any $b \in B \backslash C_{i+1}$. It follows that $C_{i+1} / / C_{i}=O_{\vartheta}\left(B / / C_{i}\right)$. Hence, $\left\{C_{i}\right\}_{i=0}^{n}$ is the radical thin chain $\mathcal{J}$ of $B$, and it is bi-anchored with length $n$.

REMARK 4.3. Regard the groups $G, C$ above as association schemes in the usual way. (For $g \in G$, the relation $g_{L}$ on underlying set $G$ is given by $(x, y) \in g_{L}$ iff $x y^{-1}=g$ 
for all $x, y \in G$; and similarly for $C$.) The adjacency algebras are isomorphic as table algebras to the group algebras $\mathbb{C} G$ and $\mathbb{C} C$, and the partial wreath product constructed above is realized as the adjacency algebra of the wedge product of the schemes [12, Section 3]. So Example 4.2 applies to association schemes.

\section{Nilpotent STAs}

We turn now to thin-central chains and nilpotent STAs. Theorems 1.20, 5.2, one direction of Theorem 5.3, and their proofs given below, are generalized directly from Hanaki's results in [9]. Again, $(A, B)$ is always a STA.

Proof of Theorem 1.20. Let $\left\{C_{i}\right\}_{i=0}^{n},\{1\} \subset C_{1} \subset \cdots \subset C_{n-1} \subset C_{n}$ be a TC-chain. Note first that $C_{1}$ is normal in $B$; and then by Proposition 2.5(v) all $C_{i}$ are normal in $B$. Now $C_{1}=C_{1} / / C_{0}$ is thin, and $C_{1} \subseteq Z(B)$. So $C_{1} \subseteq O_{\vartheta}(B) \cap Z(B)=Z_{1}$.

Suppose that $C_{i} \subseteq Z_{i}$ for some $i \geqslant 1$. Now $C_{i}$ is strongly normal in $C_{i+1}$, and $Z_{i}$ is normal in $B$. Hence, Remark 2.7 and Lemma 2.6 imply that $Z_{i}=Z_{i} C_{i}$ is strongly normal in $Z_{i} C_{i+1}$. Thus, $Z_{i} C_{i+1} / / Z_{i}$ is thin. Since $C_{i+1} / / C_{i} \subseteq Z\left(B / / C_{i}\right)$, for any $c \in C_{i+1}$ and $b \in B, c / / C_{i} \cdot b / / C_{i}=b / / C_{i} \cdot c / / C_{i}$. Hence, $c b C_{i}=b c C_{i}$, which, as $C_{i} \subseteq Z_{i}$, implies that $c b Z_{i}=b c Z_{i}$. Since $c / / Z_{i}$ is thin, $c / / Z_{i} \cdot b / / Z_{i}=y / / Z_{i}$ for any $y \in c b Z_{i}$, and similarly for $b / / Z_{i} \cdot c / / Z_{i}$. Therefore, $c / / Z_{i} \cdot b / / Z_{i}=b / / Z_{i} \cdot c / / Z_{i}$, whence $c / / Z_{i} \in Z\left(B / / Z_{i}\right)$. Then $Z_{i} C_{i+1} / / Z_{i} \subseteq O_{\vartheta}\left(B / / Z_{i}\right) \cap Z\left(B / / Z_{i}\right)=Z_{i+1} / / Z_{i}$, and so $C_{i+1} \subseteq Z_{i+1}$. The theorem follows.

Lemma 5.1. Let $(A, B)$ be a STA, and let $\mathcal{C}=\left\{C_{i}\right\}_{i=0}^{q}, C_{0} \supset C_{1} \supset \cdots \supset C_{q}$ be a $T C$-chain of length $q$ such that $C_{q}$ is a normal closed subset of $B$. Let $Q$ be a closed subset of $B$ such that $B \supseteq Q \supseteq O^{\vartheta}(B)$ and $Q / / O^{\vartheta}(B)$ is normal in $B / / O^{\vartheta}(B)$. Define $Q_{i}:=Q \cap C_{i}$ for $0 \leqslant i \leqslant q$. Then the distinct members of $\left\{Q_{i}\right\}_{i=0}^{q}$ form a $T C$-chain in $B$ of length at most $q$.

Proof. Each quotient $Q_{i} / / Q_{i+1}=\left(Q \cap C_{i}\right) / /\left(Q \cap C_{i+1}\right)$ is thin, by Theorem 4.1(i). Since $B / / O^{\vartheta}(B)$ is a group, our hypothesis that $Q / / O^{\vartheta}(B)$ is normal in $B / / O^{\vartheta}(B)$ implies that it is strongly normal. Hence, $Q$ is strongly normal in $B$ by Proposition 2.5(iii). Each $C_{i} / / C_{i+1}$ is normal in $B / / C_{i+1}$, since $C_{i} / / C_{i+1} \subseteq Z\left(B / / C_{i+1}\right)$ by the definition of a TC-chain. Since $C_{q}$ is normal in $B$, it follows from Proposition 2.5(v) that each $C_{i}$ is normal in $B$.

If $x \in Q_{i}$ and $b \in B$, then $x / / Q_{i+1}$ thin implies that $x / / Q_{i+1} \cdot b / / Q_{i+1} \cdot x^{*} / / Q_{i+1}$ is a basis element in $B / / Q_{i+1}$. Since $Q$ is strongly normal in $B$ and $x^{*} \in Q$, $\operatorname{Supp}\left(b x^{*} b^{*}\right) \subseteq Q$, hence $\operatorname{Supp}\left(x b x^{*} b^{*}\right) \subseteq Q$. Also, $x \in C_{i}$, and $C_{i} / / C_{i+1} \subseteq$ $Z\left(B / / C_{i+1}\right)$ imply that $x / / C_{i+1} \cdot b / / C_{i+1} \cdot x^{*} / / C_{i+1}=b / / C_{i+1}$. Therefore, $\operatorname{Supp}\left(x / / C_{i+1} \cdot b / / C_{i+1} \cdot x^{*} / / C_{i+1} \cdot b^{*} / / C_{i+1}\right)$ contains $1 / / C_{i+1}$. Since $C_{i+1}$ is normal in $B$, this says that $\operatorname{Supp}\left(x b x^{*} b^{*} C_{i+1}\right) \cap C_{i+1} \neq \varnothing$, hence (via the Hermitian form $), \operatorname{Supp}\left(x b x^{*} b^{*}\right) \cap C_{i+1} \neq \varnothing$. Since $Q_{i+1}=Q \cap C_{i+1}, \operatorname{Supp}\left(x b x^{*} b^{*}\right) \cap Q_{i+1} \neq$ $\varnothing$. So $1 / / Q_{i+1} \in \operatorname{Supp}\left(x / / Q_{i+1} \cdot b / / Q_{i+1} \cdot x^{*} / / Q_{i+1} \cdot b^{*} / / Q_{i+1}\right)$, and thus $x / / Q_{i+1} \cdot b / / Q_{i+1}=b / / Q_{i+1} \cdot x / / Q_{i+1}$. Therefore, $Q_{i} / / Q_{i+1} \subseteq Z\left(B / / Q_{i+1}\right)$ for all $i$. Thus, the distinct terms of $\left\{Q_{i}\right\}_{i=0}^{q}$ form a TC-chain of length at most $q$.

Proof of Theorem 1.23. Let $\mathcal{C}=\left\{C_{i}\right\}_{i=0}^{u}$ be a BTC-chain of length $u$, the nilpotence class of $(A, B)$, such that $\sum_{i=0}^{u} o\left(C_{i}\right)$ is minimal. Then $\mathcal{C}$ is immediately a lower BTC-chain. Write $\mathcal{C}$ as $B=C_{0} \supset C_{1} \supset \cdots \supset C_{u}=\{1\}$. Let $Q=O^{\alpha}(B)$. By Lemma 5.1, the distinct terms of $\left\{Q \cap C_{i}\right\}_{i=0}^{u}$ form a TC-chain of length at most $u$. Now $B / / C_{1} \subseteq Z\left(B / / C_{1}\right)$ and is thin, so $B / / C_{1}$ is an abelian group. Therefore, $C_{1} \supseteq Q$. Hence, $Q \cap C_{1}=Q=Q \cap C_{0}$. So this TC-chain starts with $Q$ and has length at most $u-1$. Since $B / / Q$ is an abelian group, we have that the distinct terms of $\{B\} \cup\left\{Q \cap C_{i}\right\}_{i=0}^{u}$ form a BTC-chain of length at most $u$. But Theorem 1.20 implies 
that the length is at least $u$; so we have that all terms are distinct and form a terminal BTC-chain of length $u$,

$$
B \supset Q=Q \cap C_{1} \supset Q \cap C_{2} \supset \cdots \supset Q \cap C_{u-1} \supset Q \cap C_{u}=\{1\} .
$$

Now $o(B)+\sum_{i=1}^{u} o\left(Q \cap C_{i}\right) \leqslant \sum_{i=0}^{u} o\left(C_{i}\right)$. But minimality of the latter implies that the sums are equal. Since $Q \cap C_{i} \subseteq C_{i}$, and hence $o\left(Q \cap C_{i}\right) \leqslant o\left(C_{i}\right)$ for $1 \leqslant i \leqslant n$, it follows that $Q \cap C_{i}=C_{i}$. Thus, $C_{1}=Q \cap C_{1}=Q$, and every lower BTC-chain of length $u$ begins $B \supset O^{\alpha}(B)$.

TheOrem 5.2. Let $(A, B)$ be a nilpotent $S T A$ of class $u$, and let $B=C_{u} \supset C_{u-1} \supset$ $\cdots \supset C_{1} \supset C_{0}=\{1\}$ be a BTC-chain of length $u$. Let $V$ be any closed subset of $B$. Then the distinct members of $\left\{V \cap C_{i}\right\}_{i=0}^{u}$ form a BTC-chain for $V$; hence $(\mathbb{C} V, V)$ is nilpotent of class at most $u$.

Proof. Each $C_{i}$ is normal in $B$, hence each $V \cap C_{i}$ is normal in $V$. By Theorem 4.1(i), $\left(V \cap C_{i+1}\right) / /\left(V \cap C_{i}\right)$ is thin for $0 \leqslant i \leqslant u-1$. Since $\left(V \cap C_{i+1}\right) C_{i} / / C_{i} \subseteq C_{i+1} / / C_{i}$, which is central in $B / / C_{i}$, then $\left(V \cap C_{i+1}\right) C_{i} / / C_{i}$ is central in $V C_{i} / / C_{i}$.

By Lemma 2.4, $V / /\left(V \cap C_{i}\right) \cong V C_{i} / / C_{i}$, where the isomorphic correspondence between table bases is $v / /\left(V \cap C_{i}\right) \leftrightarrow v / / C_{i}$ for all $v \in V$. Under this bijection, $\left(V \cap C_{i+1}\right) / /\left(V \cap C_{i}\right) \leftrightarrow\left(V \cap C_{i+1}\right) C_{i} / / C_{i}$. It follows that $\left(V \cap C_{i+1}\right) / /\left(V \cap C_{i}\right)$ is central in $V / /\left(V \cap C_{i}\right)$. Therefore, the distinct members of $\left\{V \cap C_{i}\right\}_{i=0}^{u}$ comprise a BTC-chain for $V$; and $(\mathbb{C} V, V)$ is nilpotent of class at most $u$.

Recall that the upper TC-chain $\mathcal{Z}=\left\{Z_{i}\right\}$ is defined for any STA $(A, B)$ in Definition 1.11; and is bi-anchored if and only if $(A, B)$ is nilpotent, by Theorem 1.20.

THEOREM 5.3. Let $(A, B)$ be a STA, and $D$ a closed subset of $B$. Then $(A, B)$ is nilpotent if and only if $(A / / D, B / / D)$ is nilpotent and $Z_{k} \supseteq D$ for some $k \geqslant 0$.

Proof. Suppose that $(A, B)$ is nilpotent, say of class $u$, so that $Z_{u}=B \supseteq D$. Since all the $Z_{i}$ are normal in $B$, Theorem 4.1(i) implies that the distinct terms of $\left\{D Z_{i} / / D\right\}_{i=0}^{u}$ form a BT-chain

$$
B / / D=D Z_{u} / / D \supseteq D Z_{u-1} / / D \supseteq \cdots \supseteq D Z_{1} / / D \supseteq D Z_{0} / / D=D / / D
$$

for $B / / D$. Then for all $y \in Z_{i}$ and $b \in B$,

$$
\begin{aligned}
y / / Z_{i-1} \cdot b / / Z_{i-1} & =b / / Z_{i-1} \cdot y / / Z_{i-1} \Rightarrow y b Z_{i-1}=b y Z_{i-1} \\
& \Rightarrow y b D Z_{i-1}=b y D Z_{i-1} \Rightarrow D Z_{i-1} y b D Z_{i-1}=D Z_{i-1} b y D Z_{i-1} .
\end{aligned}
$$

Now $Z_{i-1}$ normal in $B$ and $y / / Z_{i-1}$ central in $B / / Z_{i-1}$ imply that $D Z_{i-1} y=$ $Z_{i-1} y D=y D Z_{i-1}$. So

$$
D Z_{i-1} y b D Z_{i-1}=y\left(D Z_{i-1} b D Z_{i-1}\right)=\left(D Z_{i-1} y D Z_{i-1}\right)\left(D Z_{i-1} b D Z_{i-1}\right) .
$$

Similarly, $D Z_{i-1} b y D Z_{i-1}=\left(D Z_{i-1} b D Z_{i-1}\right)\left(D Z_{i-1} y D Z_{i-1}\right)$. Therefore,

$$
\left(D Z_{i-1} y D Z_{i-1}\right)\left(D Z_{i-1} b D Z_{i-1}\right)=\left(D Z_{i-1} b D Z_{i-1}\right)\left(D Z_{i-1} y D Z_{i-1}\right) .
$$

Since $y / / D Z_{i-1}$ is thin, both $y / / D Z_{i-1} \cdot b / / D Z_{i-1}$ and $b / / D Z_{i-1} \cdot y / / D Z_{i-1}$ are single basis elements in $B / / D Z_{i-1}$. So it follows from (4) that

$$
y / / D Z_{i-1} \cdot b / / D Z_{i-1}=b / / D Z_{i-1} \cdot y D Z_{i-1} \text {. }
$$

Then by Proposition 2.5(ii),

$$
\begin{aligned}
(y / / D) / /\left(D Z_{i-1} / / D\right) \cdot(b / / D) & / /\left(D Z_{i-1} / / D\right) \\
& =(b / / D) / /\left(D Z_{i-1} / / D\right) \cdot(y / / D) / /\left(D Z_{i-1} / / D\right) .
\end{aligned}
$$


Since every element of $D Z_{i} / / D$ has the form $y / / D$ for some $y \in Z_{i}$, this shows that $\left(D Z_{i} / / D\right) / /\left(D Z_{i-1} / / D\right) \subseteq Z\left((B / / D) / /\left(D Z_{i-1} / / D\right)\right)$. Therefore, the distinct terms of $\left\{D Z_{i} / / D\right\}_{i=0}^{u}$ form a BTC-chain for $B / / D$, so that $(A / / D, B / / D)$ is nilpotent.

Conversely, suppose that $(A / / D, B / / D)$ is nilpotent and $Z_{k} \supseteq D$ for some $k \geqslant$ 0 . Then $\left(A / / Z_{k}, B / / Z_{k}\right)=\left((A / / D) / /\left(Z_{k} / / D\right),(B / / D) / /\left(Z_{k} / / D\right)\right)$ is nilpotent, by Proposition 2.5(ii) and the first part of this proof. Hence, there is a TC-chain

$$
Z_{k} / / Z_{k}=Y_{0} / / Z_{k} \subset Y_{1} / / Z_{k} \subset \cdots \subset Y_{m} / / Z_{k}=B / / Z_{k} .
$$

So $Y_{i} / / Y_{i-1}=\left(Y_{i} / / Z_{k}\right) / /\left(Y_{i-1} / / Z_{k}\right)$ is thin and contained in

$$
Z\left(\left(B / / Z_{k}\right) / /\left(Y_{i-1} / / Z_{k}\right)\right)=Z\left(B / / Y_{i-1}\right),
$$

for each $1 \leqslant i \leqslant m$. Thus,

$$
\{1\}=Z_{0} \subset Z_{1} \subset \cdots \subset Z_{k} \subset Y_{1} \subset \cdots \subset Y_{m-1} \subset Y_{m}=B
$$

is a BTC-chain for $B$, and $(A, B)$ is nilpotent.

Corollary 5.4. A STA $(A, B)$ is nilpotent if and only if $B / / O^{\vartheta}(B)$ is a nilpotent group and $Z_{k} \supseteq O^{\vartheta}(B)$ for some integer $k \geqslant 0$.

EXAMPLE 5.5. Fix an odd prime $p$. Let $G$ be a $p$-group of nilpotence class $u \geqslant 3$, hence with lower central series

$$
G=G_{0} \supset G_{1}=[G, G] \supset G_{2}=\left[G, G_{1}\right] \supset \cdots \supset G_{u-2} \supset G_{u-1} \supset G_{u}=\{1\} .
$$

Assume furthermore that $G_{u-2}$ is abelian, and $\left|G_{u-1}\right|=p$. (This holds, for example, if $G$ is the multiplicative group of upper unitriangular $(u+1) \times(u+1)(u \geqslant 3)$ matrices over the field $\mathbb{F}_{p}$.) Let $H=G_{u-2} ; Y=\langle y\rangle$, a group of order $p ; C=H \times Y$, and $\psi: C \rightarrow H$ be the group homomorphism $h y^{s} \mapsto h$ for all $h \in H, y^{s} \in Y$. As in Example 4.2 , let $B$ be the standard rescaling of the partial wreath product $C \circ_{\psi} G$, and $A=\mathbb{C} B$. Then

$$
B=\left\{b_{g}:=p g \mid g \in G \backslash H\right\} \cup C,
$$

where $b_{g}\left(h y^{s}\right)=b_{g} h,\left(h y^{s}\right) b_{g}=h b_{g}$ for all $g \in G \backslash H, h \in H, y^{s} \in Y$; and if $g_{1}, g_{2} \in G \backslash H$, then

$$
b_{g_{1}} b_{g_{2}}= \begin{cases}p h Y^{+} & \text {if } g_{1} g_{2}=h \in H, \\ p b_{g_{1} g_{2}} & \text { if } g_{1} g_{2} \notin H .\end{cases}
$$

In particular, $y^{s} b_{g}=b_{g} y^{s}=b_{g}$, and $b_{g} b_{g^{-1}}=p Y^{+}$, for all $g \in G \backslash H$. Thus, $b_{g}^{*}=b_{g^{-1}}$. Now $B / / Y \cong G$ via the correspondence $b_{g} / / Y \leftrightarrow g$ for all $g \in G \backslash H$ and $h y^{s} / / Y \leftrightarrow h$ for all $h \in H, y^{s} \in Y$. We so identify the two groups.

Define

$$
Q_{i}:=\left\{b_{g} \mid g \in G_{i} \backslash H\right\} \cup C, \quad 0 \leqslant i \leqslant u-2 .
$$

Then $Q_{i}$ is a closed subset, $Q_{i} \supseteq Y$, and $Q_{i} / / Y=G_{i}$. So for $1 \leqslant i \leqslant u-2$,

$$
Q_{i-1} / / Q_{i}=\left(Q_{i-1} / / Y\right) / /\left(Q_{i} / / Y\right)=G_{i-1} / / G_{i}
$$

$$
\text { is thin and is central in } G / / G_{i}=(B / / Y) / /\left(Q_{i} / / Y\right)=B / / Q_{i} \text {. }
$$

Because $\left\{G_{i}\right\}$ is the lower central series for $G$, each $Q_{i}$, for $1 \leqslant i \leqslant u-2$, is the unique minimal closed subset in $Q_{i-1}$ among all closed subsets that contain $Y$ such that (5) holds. But any closed subset of $B$ that is not contained in $C$ contains $b_{g}$ for some $g \in G \backslash H$, and so contains $\operatorname{Supp}\left(b_{g} b_{g}^{*}\right)=Y$. Therefore, for $1 \leqslant i \leqslant u-2, Q_{i}$ is in fact the unique minimal closed subset in $Q_{i-1}$ so that (5) is true. Hence, $\sum_{i=0}^{u-2} o\left(Q_{i}\right)$ is uniquely minimal for all TC-chains of length $u-2$ that proceed down from $B$.

Let $G_{u-1}=\langle z\rangle$, of order $p$ by our choice of $G$. Fix any integer $j$ with $1 \leqslant j<p$, and let $D_{j}:=\left\langle z^{j} y\right\rangle$. Then $G_{u-1}$ central in $G$ and $Y$ central in $B$ imply that each $D_{j} \subseteq Z(B)$. Since $C$ is an abelian group (as $H=G_{u-2}$ is abelian, again by choice 
of $G), Q_{u-2} / / D_{j}=C / / D_{j}$ is thin and abelian. Furthermore, for all $x=h y^{r} \in Q_{u-2}$ and $b_{g} \in B \backslash Q_{u-2}$, we have in the nilpotent group $G$ that $h g=g h z^{t}$ for some integer $t$. So in $B, b_{g}=p g, y^{r} b_{g}=b_{g} y^{r}=b_{g}$, and $C$ abelian yield

$$
x b_{g}=h y^{r} b_{g}=h b_{g}=b_{g} h z^{t}=b_{g} h y^{r} z^{t}=b_{g} x z^{t}=b_{g} z^{t} x .
$$

Now $z^{t}=z^{j s}$ for some integer $s$, and $b_{g} z^{j s}=b_{g}\left(z^{j} y\right)^{s}$. Hence,

$$
x b_{g} D_{j}=b_{g} x\left(z^{j} y\right)^{s} D_{j}=b_{g} x D_{j} .
$$

Therefore, $x / / D_{j} \cdot b_{g} / / D_{j}=b_{g} / / D_{j} \cdot x / / D_{j}$, thus $Q_{u-2} / / D_{j} \subseteq Z\left(B / / D_{j}\right)$. Since $D_{j} \subseteq Z(B)$ and $o\left(D_{j}\right)=p$, it follows that for each $1 \leqslant j<p$,

$$
B \supset Q_{1} \supset Q_{2} \supset \cdots \supset Q_{u-2} \supset D_{j} \supset\{1\}
$$

is a lower BTC-chain for $B$. Each $Q_{i}$ is the unique minimal closed subset of $Q_{i-1}$ that exists in a BTC-chain, for all $i \leqslant u-2$; but each $D_{j}$ is minimal such in $Q_{u-2}$. Thus there are $p-1$ stringently minimal such chains, not a unique one. As in Example 4.2, the algebraic construction here is realized as the adjacency algebra of the wedge product of association schemes. Hence this example too applies to association schemes. So the answer to [9, Question 2.11] is negative.

Our final result displays the role played in general by the subsets $\operatorname{Supp}\left(b^{*} b\right)$ for $b \in B$ in finding TC-chains in $B$ from the top down, with each term minimal in the previous one.

THeOREM 5.6. Let $U$ be a closed subset in a STA $(A, B)$. Let $V=O^{\alpha}(U)$. Let $S$ be the closed subset of $U$ such that $S \supseteq V$ and $S / / V=\left\langle\operatorname{Supp}\left(b^{*} / / V \cdot b / / V\right) \cap(U / / V)\right|$ $b \in B\rangle$. Assume that $U / / V$ is normal in $B / / V$. Then the following hold:

(i) There is a unique closed subset $C$ of $U$ that is minimal (with respect to inclusion) such that $C \supseteq S, C / / V$ is normal in $B / / V$, and $U / / C \subseteq Z(B / / C)$.

(ii) If $D$ is any closed subset of $U$ that is minimal with respect to inclusion such that $V \subseteq D, D / / V$ is normal in $B / / V$, and $U / / D \subseteq Z(B / / D)$, then $D \subseteq C$.

Proof. If $Y$ is any closed subset with $V \subseteq Y \subseteq U$, then $U / / V$ an abelian group implies that $Y / / V$ is a normal abelian subgroup of $U / / V$. Hence by $V$ normal in $U$ and Proposition 2.5(v), $Y$ is normal in $U$; and $U / / Y$ is an abelian group, in particular is thin.

Suppose that $C$ is a closed subset of $U$ such that $V \subseteq C, C / / V$ is normal in $B / / V$, and $U / / C \subseteq Z(B / / C)$. Then $U / / C$ thin implies that for any $x \in U$ and $b \in B$, $x / / C \cdot b / / C \cdot x^{*} / / C=b / / C$. Hence,

$$
(x / / V) / /(C / / V) \cdot(b / / V) / /(C / / V) \cdot\left(x^{*} / / V\right)(C / / V)=(b / / V) / /(C / / V),
$$

which, since $C / / V$ is thin and normal in $B / / V$, implies that

$$
x / / V \cdot b / / V \cdot x^{*} / / V=b / / V \cdot c_{x, b} / / V, \text { some } c_{x, b} \in C .
$$

Suppose that $D$ is another closed subset of $U$ with $V \subseteq D, D / / V$ normal in $B / / V$, and $U / / D \subseteq Z(B / / D)$. Since $V \subseteq C \cap D \subseteq U, U / / C \cap D$ is also thin. An argument similar to the one above yields that for all $x \in U$ and $b \in B$,

$$
x / / V \cdot b / / V \cdot x^{*} / / V=b / / V \cdot d_{x, b} / / V, \text { some } d_{x, b} \in D .
$$

Therefore, $b / / V \cdot c_{x, b} / / V=b / / V \cdot d_{x, b} / / V$, so that $b / / V \cdot\left(c_{x, b} / / V\right) \cdot\left(d_{x, b} / / V\right)^{-1}=$ $b / / V$. Then the thin element $\left(c_{x, b} / / V\right) \cdot\left(d_{x, b} / / V\right)^{-1}$ is in $\operatorname{Supp}\left(b^{*} / / V \cdot b / / V\right)$, by the Hermitian form for the STA $B / / V$. Thus, $\left(c_{x, b} / / V\right) \cdot\left(d_{x, b} / / V\right)^{-1} \in S / / V$. 
Suppose that $C \supseteq S$. Then $d_{x, b} / / V \in(C / / V) \cdot\left(c_{x, b} / / V\right)=C / / V$. Therefore, $d_{x, b} \in C \cap D$. Now $V \subseteq C \cap D$, and both closed subsets are normal in $U$. Hence,

$$
\begin{aligned}
x / / V \cdot b / / V \cdot x^{*} / / V=b / / V \cdot d_{x, b} / / V & \\
\quad \Rightarrow & V x b x^{*} V=V b d_{x, b} V \\
\Rightarrow & (C \cap D) x b x^{*}(C \cap D)=(C \cap D) b d_{x, b}(C \cap D) \\
\Rightarrow & (x / / C \cap D) \cdot(b / / C \cap D) \cdot\left(x^{*} / / C \cap D\right)=(b / / C \cap D) \cdot\left(d_{x, b} / / C \cap D\right) \\
& =b / / C \cap D,
\end{aligned}
$$

since $d_{x, b} \in C \cap D$ implies that $d_{x, b} / / C \cap D=1 / / C \cap D$. It follows that $U / /(C \cap D) \subseteq$ $Z(B / /(C \cap D))$. Now $C / / V$ and $D / / V$ are normal in $B / / V$. So if $x \in C \cap D$ and $b \in B$, then

$$
x / / V \cdot b / / V=b / / V \cdot c / / V=b / / V \cdot d / / V
$$

for some $c \in C, d \in D$ (both of which depend on $x$ and $b$ ). Hence, $b / / V \cdot c / / V$. $(d / / V)^{-1}=b / / V$, so that

$$
c / / V \cdot(d / / V)^{-1} \in \operatorname{Supp}\left(b^{*} / / V \cdot b / / V\right) \cap(U / / V) \subseteq S / / V \subseteq C / / V .
$$

Therefore, $d / / V \in C / / V$, so $d \in C \cap D$. We now have that $((C \cap D) / / V) \cdot(b / / V) \subseteq$ $(b / / V) \cdot((C \cap D) / / V)$. Replacing $b$ by $b^{*}$ and then applying the anti-automorphism yields the opposite containment, hence $(C \cap D) / / V$ is normal in $B / / V$.

We have shown that $C \cap D$ satisfies the same hypotheses as $D$, provided that $C \supseteq S$. Both claims of the theorem follow immediately.

\section{REFERENCES}

[1] Zvi Arad, Elsa Fisman, and Mikhail Muzychuk, Generalized table algebras, Israel J. Math. 114 (1999), 29-60.

[2] Harvey I. Blau, Quotient structures in C-algebras, J. Algebra 177 (1995), no. 1, 297-337.

[3] _ Table algebras, European J. Combin. 30 (2009), no. 6, 1426-1455.

[4] _ Fusion rings with few degrees, J. Algebra 396 (2013), 220-271.

[5] Harvey I. Blau and Shengan Chen, Normal series and character values in p-standard table algebras, Comm. Algebra 45 (2017), no. 11, 4646-4655.

[6] Harvey I. Blau and Paul-Hermann Zieschang, Sylow theory for table algebras, fusion rule algebras, and hypergroups, J. Algebra 273 (2004), no. 2, 551-570.

[7] Christopher French and Paul-Hermann Zieschang, On residually thin hypergroups, J. Algebra 551 (2020), 93-118.

[8] Shlomo Gelaki and Dmitri Nikshych, Nilpotent fusion categories, Adv. Math. 217 (2008), no. 3, 1053-1071.

[9] Akihide Hanaki, Nilpotent schemes and group-like schemes, J. Combin. Theory Ser. A 115 (2008), no. 2, 226-236.

[10] Akihide Hanaki and Izumi Miyamoto, Classification of association schemes with small vertices, http://math.shinshu-u.ac.jp/ hanaki/as/, 2021.

[11] Akihide Hanaki and Osamu Shimabukuro, Indecomposable decompositions of modular standard modules for two families of association schemes, J. Algebraic Combin. 46 (2017), no. 2, 445-453.

[12] Mikhail Muzychuk, A wedge product of association schemes, European J. Combin. 30 (2009), no. 3, 705-715.

[13] Paul-Hermann Zieschang, An algebraic approach to association schemes, Lecture Notes in Mathematics, vol. 1628, Springer-Verlag, Berlin, 1996

[14] _ Theory of association schemes, Springer Monographs in Mathematics, Springer-Verlag, Berlin, 2005.

Harvey I. Blau, Department of Mathematical Sciences, Northern Illinois University, DeKalb, IL 60115, USA

E-mail : hblau@niu.edu 